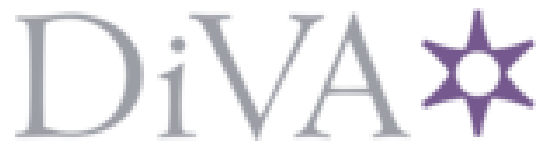

http://www.diva-portal.org

Postprint

This is the accepted version of a paper presented at 49th IEEE Conference on Decision and Control (CDC). Atlanta, GA. DEC 15-17, 2010.

Citation for the original published paper:

Zaidi, A A., Oechtering, T J., Skoglund, M. (2010)

Sufficient Conditions for Closed-Loop Control Over Multiple-Access and Broadcast Channels

In: 49TH IEEE CONFERENCE ON DECISION AND CONTROL (CDC) (pp. 4771-4776). https://doi.org/10.1109/CDC.2010.5717279

N.B. When citing this work, cite the original published paper.

Permanent link to this version:

http://urn.kb.se/resolve?urn=urn:nbn:se:kth:diva-52391 


\title{
Sufficient Conditions for Closed-Loop Control Over Multiple-Access and Broadcast Channels
}

\author{
Ali A. Zaidi, Tobias J. Oechtering and Mikael Skoglund
}

\begin{abstract}
The problem of closed-loop stabilization of two scalar linear time invariant systems over noisy multiple-access and broadcast communication channels with arbitrarily distributed initial states is addressed. We propose to use communication and control schemes based on the coding schemes introduced by Ozarow et al. for the multiple-access and the broadcast channels with noiseless feedback which are extensions of the Schalkwijk-Kailath coding scheme. By employing the proposed communication and control schemes over the multipleaccess and the broadcast channels, we derive stability regions those are sufficient for mean square stability of the two linearly controlled LTI systems.
\end{abstract}

\section{INTRODUCTION}

The problem of remotely controlling dynamical systems over communication channels has gained significant attention in recent years. Such problems ask for interaction between stochastic control theory and information theory [1,2]. The minimum data rate below which the stability of an LTI system is impossible has been derived in stochastic and deterministic settings in [3-5], where they considered quantization errors and noise-free rate-limited channels. In [6,7] are necessary rate conditions required to stabilize an LTI plant almost surely. However, from [8] we know that in general the characterization by Shannon capacity is not enough for sufficient conditions for moment stability in closed-loop control. In [9] a simple coding scheme is proposed to mean square stabilize an LTI plant over noise-free rate-limited channels. The mean square stability of discrete plant over signal-to-noise ratio constraint channels is addressed in [10, 11]. In [12] the authors considered noisy communication links between both observer-controller and controller-plant.

We formulate the general problem of controlling two scalar linear time invariant systems over the white Gaussian multiple-access and broadcast channels. The two user multiple-access channel is the communication channel where two sources transmit their messages to a common destination [13]. By the control over the multiple-access channel we mean that there exist two separate sensors to sense the states and a single remote controller to stabilize the two plants i.e., a multi-sensor joint controller setup. The capacity region of the two-user memoryless Gaussian multiple-access channel with noiseless feedback is found in [14], which is relevant to the problem of closed-loop control over the multiple-access

This work was supported in part by the European Commission through the FP7 project FeedNetBack (co-design for networked control systems).

Ali A. Zaidi, Tobias J. Oechtering and Mikael Skoglund are with the School of Electrical Engineering and the ACCESS Linnaeus Center, Royal Institute of Technology (KTH), Stockholm, Sweden. channel. The two user broadcast channel is the communication channel where one sender transmits messages to two destinations [13]. Control over broadcast channel refers to a joint sensor multi-controller setup i.e., there exists a common sensor to jointly observe the states of the two plants and there are two separate remote controllers in order to stabilize them. The capacity region of the Gaussian broadcast channel with and without feedback is not known [13]. For the problem of closed-loop control, the broadcast channel with feedback is more relevant. In [15] Ozarow et al. provided an achievable rate region over the two user memoryless Gaussian broadcast channel with noiseless feedback which is highly relevant to our problem. The coding schemes proposed by Ozarow et al. in $[14,15]$ for the memoryless Gaussian multiple-access and the broadcast channels with noiseless are extensions of the well-known Schalkwijk-Kailath coding scheme for memoryless Gaussian point-to-point communication channel with noiseless feedback [16].

We used the Schalkwijk-Kailath type scheme for deriving rate sufficient conditions for closed-loop stabilization of a scalar LTI sytem over white Gaussian relay channels in [17, 18]. In this paper we consider an extension to two scalar LTI plants with arbitrary distributed initial states which have to be remotely stabilized over the white Gaussian multipleaccess and the white Gaussian broadcast channels. We use linear and memoryless communication and control schemes based on Ozarow's coding schemes to derive regions which are sufficient for mean square stability $[3,4,8-10,17]$ of the two plants in the absence of process and measurement noises.

\section{Problem SetuP}

We consider two scalar discrete-time LTI systems whose state equations are given by

$$
\begin{aligned}
X_{i, t+1} & =\lambda_{i} X_{i, t}+U_{i, t}+W_{i, t} \\
Y_{i, t} & =X_{i, t}+V_{i, t} \quad \text { for } \quad i=1,2
\end{aligned}
$$

where $\left\{X_{i, t}\right\} \subseteq \mathbb{R},\left\{U_{i, t}\right\} \subseteq \mathbb{R},\left\{Y_{i, t}\right\} \subseteq \mathbb{R},\left\{W_{i, t}\right\} \subseteq \mathbb{R}$, and $\left\{V_{i, t}\right\} \subseteq \mathbb{R}$ are state, control, observation, process noise and measurement noise processes of the plant $i$. We assume that the open-loop systems are unstable $\left(\lambda_{i}>1\right)$ and the initial states $X_{i, 0}$ are random variables with arbitrary probability distributions having variance $\alpha_{i, 0}=\mathbb{E}\left[X_{i, 0}^{2}\right]$ and correlation coefficient $\rho_{0}=\frac{\mathbb{E}\left[X_{1,0} X_{2,0}\right]}{\sqrt{\alpha_{1,0} \alpha_{2,0}}}$. We study the problem of remotely controlling the two unstable systems over the white Gaussian broadcast and multiple-access channels. 


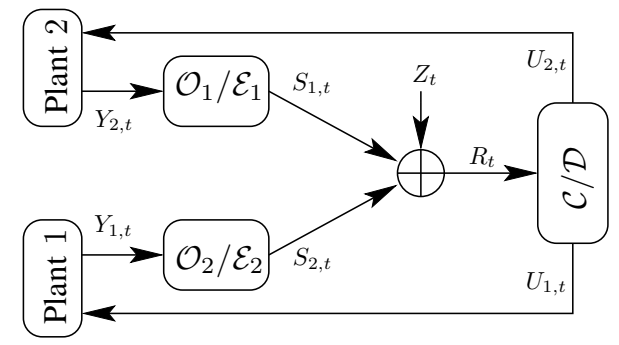

Fig. 1. The two unstable LTI plants have to be controlled over the white Gaussian multiple access channel. There are two sensors to separately sense the states of the two plants and there is a remote common control unit.

\section{A. Control over multiple-access channel}

The setup for control over multiple-access channel is depicted in Fig. 1. There are separate observers $\mathcal{O}_{1}$ and $\mathcal{O}_{2}$ for the two plants, and there is a common control unit $\mathcal{C}$ situated at remote location. In order to communicate the observed state values to the controller, an encoder $\mathcal{E}_{i}$ is lumped with $\mathcal{O}_{i}$ and a decoder $\mathcal{D}$ is lumped with the controller. At any time instant $t$, the encoders $\mathcal{E}_{1}$ and $\mathcal{E}_{2}$ transmit $S_{1, t}$ and $S_{2, t}$ respectively, and the decoder $\mathcal{D}$ receives $R_{t}=S_{1, t}+S_{2, t}+$ $Z_{t}$, where $Z_{t} \sim \mathcal{N}(0, N)$ is the white noise component. Let $f_{i, t}$ denote the observer/encoder policy for the plant $i$, then we have $S_{i, t}=f_{i, t}\left(\left\{Y_{i, k}\right\}_{k=0}^{t}\right)$ which must satisfy an average power constraint $\lim _{T \rightarrow \infty} \frac{1}{T} \sum_{t=0}^{T-1} \mathbb{E}\left[S_{i, t}^{2}\right] \leq P_{i}$. Further let $\gamma_{i, t}$ denote the decoder/controller policy, then $U_{i, t}=\gamma_{i, t}\left(\left\{R_{k}\right\}_{k=0}^{t}\right)$.

\section{B. Control over broadcast channel}

The setup for control over broadcast channel is depicted in Fig. 2. There is a common observer $\mathcal{O}$ and separate controllers $\mathcal{C}_{1}$ and $\mathcal{C}_{2}$ for the two plants. In order to communicate the observed state values to the controllers, an encoder $\mathcal{E}$ is lumped with the observer and the decoders $\mathcal{D}_{1}$ and $\mathcal{D}_{2}$ are lumped with the respective controllers. At any time instant $t$, the encoder transmits $S_{t}$ and the decoder $\mathcal{D}_{i}$ receives $R_{i, t}=S_{t}+Z_{t}+Z_{i, t}$, where $Z_{i, t} \sim \mathcal{N}\left(0, N_{i}\right)$ and $Z_{t} \sim \mathcal{N}(0, N)$ are the mutually independent white noise components. The noise component $Z_{t}$ in the broadcast channel can model a common noise or interference in the two signals. Let $f_{t}$ denote the observer/encoder policy, then we have $S_{t}=f_{t}\left(\left\{Y_{1, k}\right\}_{k=0}^{t},\left\{Y_{2, k}\right\}_{k=0}^{t}\right)$ which must satisfy an average power constraint $\lim _{T \rightarrow \infty} \frac{1}{T} \sum_{t=0}^{T-1} \mathbb{E}\left[S_{t}^{2}\right] \leq P$. Further let $\gamma_{i, t}$ denote the decoder/controller policy, then $U_{i, t}=\gamma_{i, t}\left(\left\{R_{i, k}\right\}_{k=0}^{t}\right)$.

\section{Mean square stability}

We assume that the process noise $W_{i, t}$ and the measurement noise $V_{i, t}$ in (1) are zero, and focus on mean square stability $[3,4,8,9,19]$ of the two plants. For a noise-free plant, we define mean square stability as follows.

Definition 1: A system is said to be mean square stable if and only if

$$
\lim _{t \rightarrow \infty} \mathbb{E}\left[X_{t}^{2}\right]=0,
$$

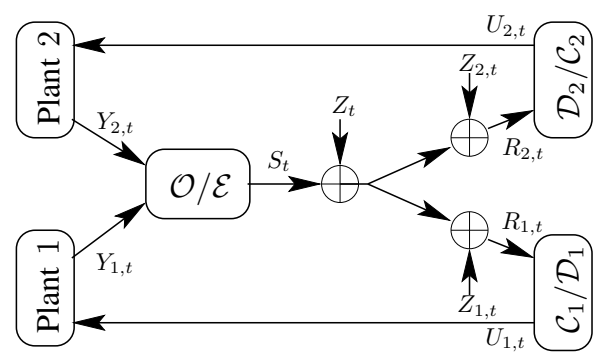

Fig. 2. The two unstable LTI plants have to be controlled over the white Gaussian broadcast channel. There is a common sensor to jointly sense the states of the two plants and there are remotely located separate control units.

regardless of the initial state $X_{0}$.

\section{MAIN RESULTS}

We will first present our results in a comprehensive fashion and then provide the proofs in the next section.

\section{A. Stability results for the multiple-access channel}

Theorem 2: The two scalar LTI systems in (1) with $W_{i, t}=V_{i, t}=0$ can be mean square stabilized over the memoryless white Gaussian multiple access channel if the systems' parameters $\left\{\lambda_{1}, \lambda_{2}\right\}$ satisfy the following inequalities

$$
\begin{aligned}
& \log \left(\lambda_{1}\right)<\frac{1}{2} \log \left(1+\frac{P_{1}\left(1-\rho^{\star 2}\right)}{N}\right), \\
& \log \left(\lambda_{2}\right)<\frac{1}{2} \log \left(1+\frac{P_{2}\left(1-\rho^{\star 2}\right)}{N}\right),
\end{aligned}
$$

where $\rho^{\star}$ is the root in the open interval $(0,1)$ of the following fourth order polynomial

$$
\begin{aligned}
\left(P_{1}\left(1-\rho^{2}\right)+N\right) & \left(P_{2}\left(1-\rho^{2}\right)+N\right)= \\
& \left(P_{1}+P_{2}+2 \rho \sqrt{P_{1} P_{2}}+N\right) N .
\end{aligned}
$$

Proof: The proof is given in Sec. IV-A.

Remark 3: It can be shown that for fully correlated initial states, i.e., $\rho_{0}=1$, the stability conditions are given by

$$
\log \left(\lambda_{i}\right)<\frac{1}{2} \log \left(1+\frac{P_{1}+P_{2}+2 \sqrt{P_{1} P_{2}}}{N}\right), \quad i=1,2 .
$$

Remark 4: The terms on the right hand side in (2) correspond to the sum-rate optimal achievable rate pair for the two sources over the white Gaussian multiple-access channel with noiseless feedback [14]. The stability region in (2) is smaller than the capacity region in [14]. This is because to ensure second moment stability the coding scheme has to have at least double exponential error decay.

\section{B. Stability results for the broadcast channel}

In the broadcast channel there is a joint encoder with an output power constraint contrary to the multiple-access channel where the two encoders have individual power constraints. Therefore the joint encoder in the broadcast 
channel has freedom to tradeoff between the powers allocated to the transmission of the observed states of the two plants.

Theorem 5: The two scalar LTI systems in (1) with $W_{i, t}=V_{i, t}=0$ can be mean square stabilized over the memoryless white Gaussian broadcast channel if the systems' parameters $\left\{\lambda_{1}, \lambda_{2}\right\}$ satisfy the following inequalities

$$
\begin{aligned}
\log \left(\lambda_{1}\right) & <\frac{1}{2} \log \left(\frac{D^{\star}\left(N+N_{1}+P\right)}{D^{\star}\left(N+N_{1}\right)+g^{2} P\left(1-\rho^{\star}\right)}\right), \\
\log \left(\lambda_{2}\right) & <\frac{1}{2} \log \left(\frac{D^{\star}\left(N+N_{2}+P\right)}{D^{\star}\left(N+N_{2}\right)+P\left(1-\rho^{\star}\right)}\right),
\end{aligned}
$$

where $D^{\star}=1+g^{2}+2 g \rho^{\star}, g \geq 0$, and $\rho^{\star}$ is the largest root in the open interval $(0,1)$ of the following polynomial

$$
\begin{aligned}
& \rho=-\left(D\left(N \Sigma+N_{1} N_{2}\right) \rho-g P \Sigma\left(1-\rho^{2}\right)\right) \times \\
& \left(\Pi\left(D\left(N+N_{1}\right)+g^{2} P\left(1-\rho^{2}\right)\right)\left(D\left(N+N_{2}\right)+g^{2} P\left(1-\rho^{2}\right)\right)\right)
\end{aligned}
$$

where $\Pi=\left(P+N+N_{1}\right)\left(P+N+N_{2}\right)$ and $\Sigma=P+N+$ $N_{1}+N_{2}$.

Proof: The proof is given in Sec. IV-B.

Remark 6: The terms on the right hand side in (4) is an achievable rate pair for the two decoders over the white Gaussian broadcast channel with noiseless feedback [15].

Remark 7: If the noise components $Z_{1, t}$ and $Z_{2, t}$ are zero in the broadcast channel model, then the two controllers receive the same signal and this setup is equivalent to having a joint controller. Therefore the stability region for the jointsensor joint-controller case can be obtained by setting $N_{1}=$ $N_{2}=0$ in (4).

The parameter $g$ in (4) can tradeoff between the stabilizability of the two plants and thus we can obtain a stability region for the given channel parameters by increasing $g$ from zero to less than infinity. Fig. 3 shows some examples of stability regions for $P=10$. The solid line shows the boundary of the stability region when $N=0$ and $N_{1}=$ $N_{2}=1$, the dashed line shows the boundary of the stability region when $N=N_{1}=N_{2}=0.5$, and the dotted line shows the boundary of the stability region when $N=1$ and $N_{1}=N_{2}=0$. In these examples $N+N_{i}=1$, and we can observe that the individual noise components $\left\{Z_{1, t}, Z_{2, t}\right\}$ are less harmful than the common noise component $Z_{t}$ due to diversity effect. For comparison we also show the stability region when the encoder separately serves the two plants in alternate time steps, i.e., in each time step there is a point-to-point communication link from the encoder to one of the controllers. For this case the necessary and sufficient conditions for mean square stability can be found in [17], which are given by

$$
\log \left(\lambda_{i}\right)<\frac{1}{4} \log \left(1+\frac{P}{N+N_{i}}\right) \quad \text { for all } i \in\{1,2\} .
$$

The boundary of the rectangular stability region defined by the above inequalities is shown in Fig. 3 for $P=10$ and $N+N_{i}=1$.

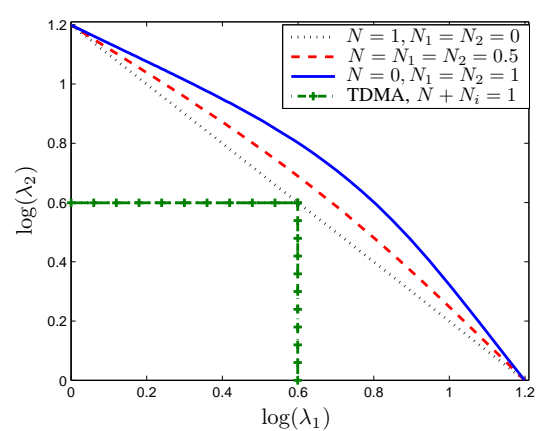

Fig. 3. Illustration of the stability regions for the broadcast channel.

\section{PROOFS}

In order to prove Theorems 2 and 5, we propose to use $-\frac{1}{2}$ coding schemes in [14] and [15]. These schemes are based on Schalkwijk-Kailath coding scheme [16]. By employing the proposed coding schemes over the given broadcast and multiple-access channels, we then find conditions on the system parameters $\left\{\lambda_{1}, \lambda_{2}\right\}$ which are sufficient to mean square stabilize the systems in (1).

\section{A. Proof of Theorem 2}

The scheme for the white Gaussian multiple-access channel works as follows.

Initial time steps, $t=0,1$ : Initially the two encoders transmit the observed state values in alternate time slots to the respective controllers. The first two disjoint transmissions in time make the plant states Gaussian distributed regardless of the distribution of their initial states, which will be explained shortly. However if the initial states are already Gaussian, then the following disjoint initial transmissions are not needed.

At time step $t=0$, the encoder $\mathcal{E}_{1}$ observes $X_{1,0}$ and transmits $S_{1,0}=\sqrt{\frac{P_{1}}{\alpha_{1,0}}} X_{1,0}$. The encoder $\mathcal{E}_{2}$ stays quiet, i.e., $S_{2,0}=0$. The decoder $\mathcal{D}$ receives $R_{0}=S_{1,0}+Z_{0}$. It then estimates $X_{1,0}$ as

$$
\hat{X}_{1,0}=\sqrt{\frac{\alpha_{1,0}}{P_{1}}} R_{0}=X_{1,0}+\sqrt{\frac{\alpha_{1,0}}{P_{1}}} Z_{0} .
$$

The controller $\mathcal{C}$ then takes an action $U_{1,0}=-\lambda_{1} \hat{X}_{1,0}$ for the plant 1 , which results in $X_{1,1}=\lambda_{1}\left(X_{1,0}-\hat{X}_{1,0}\right)$. The state $X_{1,1} \sim \mathcal{N}\left(0, \alpha_{1,1}\right)$ with $\alpha_{1,1}=\lambda_{1}^{2} \frac{\alpha_{1,0} N}{P_{1}}$. The controller does not take any action for the plant 2 , therefore $X_{2,1}=$ $\lambda_{2} X_{2,0}$ with $\alpha_{2,1}=\lambda_{2}^{2} \alpha_{2,0}$.

At time step $t=1$, the encoder $\mathcal{E}_{1}$ stays quiet. The encoder $\mathcal{E}_{2}$ observes $X_{2,1}$ and transmits $S_{2,1}=\sqrt{\frac{P_{2}}{\alpha_{2,1}}} X_{2,1}$. The decoder $\mathcal{D}$ receives $R_{1}=S_{2,1}+Z_{1}$. It then estimates $X_{2,1}$ as

$$
\hat{X}_{2,1}=\sqrt{\frac{\alpha_{2,1}}{P_{2}}} R_{1}=X_{2,1}+\sqrt{\frac{\alpha_{2,1}}{P_{2}}} Z_{1} .
$$

The controller $\mathcal{C}$ then takes an action $U_{2,1}=-\lambda_{2} \hat{X}_{2,1}$ for the plant 2, which results in $X_{2,2}=\lambda_{2}\left(X_{2,1}-\hat{X}_{2,1}\right)$. The state $X_{2,2} \sim \mathcal{N}\left(0, \alpha_{2,2}\right)$. For the plant 1 , the controller does 
not take any action $U_{1,1}=0$, therefore $X_{1,2}=\lambda_{1} X_{1,1}$ and $X_{1,2} \sim \mathcal{N}\left(0, \alpha_{1,2}\right)$.

It is noteworthy that due to non-overlapping initial transmissions by the two encoders, the states $X_{1,2}$ and $X_{2,2}$ are now zero mean Gaussian variables with correlation coefficient $\rho_{2}=\frac{\mathbb{E}\left[X_{1,2} X_{2,2}\right]}{\sqrt{\alpha_{1,2} \alpha_{2,2}}}$ equal to zero ${ }^{1}$. Henceforth the two encoders will transmit their signals simultaneously.

Further time steps $t \geq 2$ : The two encoders $\mathcal{E}_{1}$ and $\mathcal{E}_{2}$ observe $X_{1, t}$ and $X_{2, t}$, and they respectively transmit

$$
S_{1, t}=\sqrt{\frac{P_{1}}{\alpha_{1, t}}} X_{1, t}, \quad S_{2, t}=\sqrt{\frac{P_{2}}{\alpha_{2, t}}} X_{2, t} \operatorname{sgn}\left(\rho_{t}\right),
$$

where $\rho_{t}=\frac{\mathbb{E}\left[\left(X_{1, t}-\mathbb{E}\left[X_{1, t}\right]\right)\left(X_{2, t}-\mathbb{E}\left[X_{2, t}\right]\right)\right]}{\sqrt{\alpha_{1, t} \alpha_{2, t}}}$ and $\operatorname{sgn}\left(\rho_{t}\right)=1$ if $\rho_{t} \geq 0$ and $\operatorname{sgn}\left(\rho_{t}\right)=-1$ if $\rho_{t}<0$.

The decoder $\mathcal{D}$ receives $R_{t}=S_{1, t}+S_{2, t}+Z_{t}$. It then computes an MMSE estimate of the state of the plant $i$ as

$$
\hat{X}_{i, t}=\mathbb{E}\left[X_{i, t} \mid R_{t}\right] \stackrel{(a)}{=} \frac{\mathbb{E}\left[R_{t} X_{i, t}\right]}{\mathbb{E}\left[R_{t}^{2}\right]} R_{t},
$$

where $(a)$ follows from the fact that the optimum MMSE of the Gaussian variable is linear [20]; and we have

$$
\begin{aligned}
\mathbb{E}\left[X_{1, t} R_{t}\right] & =\sqrt{\alpha_{1, t}}\left(\sqrt{P_{1}}+\sqrt{P_{2}}\left|\rho_{t}\right|\right), \\
\mathbb{E}\left[X_{2, t} R_{t}\right] & =\sqrt{\alpha_{2, t}}\left(\sqrt{P_{2}}+\sqrt{P_{1}}\left|\rho_{t}\right|\right) \operatorname{sgn}\left(\rho_{t}\right), \\
\mathbb{E}\left[R_{t}^{2}\right] & =P_{1}+P_{2}+2\left|\rho_{t}\right| \sqrt{P_{1} P_{2}}+N .
\end{aligned}
$$

The controller $\mathcal{C}$ takes an action $U_{i, t}=-\lambda_{i} \hat{X}_{i, t}$ for the plant $i$, which results in $X_{i, t+1}=\lambda_{i}\left(X_{i, t}-\hat{X}_{i, t}\right)$. The mean values of the states are

$$
\begin{aligned}
\mathbb{E}\left[X_{i, t}\right] & =\mathbb{E}\left[\lambda_{i}\left(X_{i, t}-\hat{X}_{i, t}\right)\right] \\
& \stackrel{(a)}{=} \lambda_{i} \mathbb{E}\left[X_{i, t}-\frac{\mathbb{E}\left[R_{t} X_{i, t}\right]}{\mathbb{E}\left[R_{t}^{2}\right]} R_{t}\right] \stackrel{(b)}{=} 0,
\end{aligned}
$$

where ( $a$ ) follows from (6); and ( $b$ ) follows from $\mathbb{E}\left[X_{i, 2}\right]=0$ and by recursively using $(a)$. The variance of the state $X_{i, t+1}$ is given by

$$
\begin{aligned}
\alpha_{i, t+1} & \triangleq \mathbb{E}\left[X_{i, t+1}^{2}\right]=\lambda_{i}^{2} \mathbb{E}\left[\left(X_{i, t}-\hat{X}_{i, t}\right)^{2}\right] \\
& =\lambda_{i}^{2} \mathbb{E}\left[\left(X_{i, t}-\frac{\mathbb{E}\left[R_{t} X_{i, t}\right]}{\mathbb{E}\left[R_{t}^{2}\right]} R_{t}\right)^{2}\right] \\
& =\lambda_{i}^{2}\left(\mathbb{E}\left[X_{i, t}^{2}\right]-\frac{\left(\mathbb{E}\left[R_{t} X_{i, t}\right]\right)^{2}}{\mathbb{E}\left[R_{t}^{2}\right]}\right) .
\end{aligned}
$$

By using (7) in (9) we get the following recursive equations

$$
\begin{aligned}
& \alpha_{1, t+1}=\alpha_{1, t} \lambda_{1}^{2}\left(\frac{N+P_{2}\left(1-\rho_{t}^{2}\right)}{P_{1}+P_{2}+2\left|\rho_{t}\right| \sqrt{P_{1} P_{2}}+N}\right) \\
& \alpha_{2, t+1}=\alpha_{2, t} \lambda_{2}^{2}\left(\frac{N+P_{1}\left(1-\rho_{t}^{2}\right)}{P_{1}+P_{2}+2\left|\rho_{t}\right| \sqrt{P_{1} P_{2}}+N}\right)
\end{aligned}
$$

\footnotetext{
${ }^{1}$ The states in the second time step become uncorrelated irrespective of the value of the correlation between the initial states. This scheme does not exploit correlation between the initial states and thus the stability region obtained is independent of the correlation of the initial states.
}

The cross-correlation between the states is given by

$$
\begin{aligned}
& \mathbb{E}\left[X_{1, t+1} X_{2, t+1}\right]=\mathbb{E}\left[\lambda_{1}\left(X_{1, t}-\hat{X}_{1, t}\right) \lambda_{2}\left(X_{2, t}-\hat{X}_{2, t}\right)\right] \\
& \stackrel{(a)}{=} \lambda_{1} \lambda_{2}\left(\mathbb{E}\left[X_{1, t} X_{2, t}\right]-\frac{\mathbb{E}\left[X_{1, t} R_{t}\right] \mathbb{E}\left[X_{2, t} R_{t}\right]}{\mathbb{E}\left[R_{t}^{2}\right]}\right) \\
& \stackrel{(b)}{=} \lambda_{1} \lambda_{2} \sqrt{\alpha_{1, t} \alpha_{2, t}}\left(\frac{N \rho_{t}-\operatorname{sgn}\left(\rho_{t}\right) \sqrt{P_{1} P_{2}}\left(1-\rho_{t}^{2}\right)}{P_{1}+P_{2}+2\left|\rho_{t}\right| \sqrt{P_{1} P_{2}}+N}\right),
\end{aligned}
$$

where $(a)$ follows from $\mathbb{E}\left[\hat{X}_{1, t} X_{2, t}\right]=\mathbb{E}\left[\hat{X}_{2, t} X_{1, t}\right]=$ $\mathbb{E}\left[\hat{X}_{1, t} \hat{X}_{2, t}\right]=\frac{\mathbb{E}\left[X_{1, t} R_{t}\right] \mathbb{E}\left[X_{1, t} R_{t}\right]}{\mathbb{E}\left[R_{t}^{2}\right]}$; and (b) follows from (7). The correlation coefficient is then given by

$$
\begin{aligned}
& \rho_{t+1}=\frac{\mathbb{E}\left[X_{1, t+1} X_{2, t+1}\right]}{\sqrt{\alpha_{1, t} \alpha_{2, t}}} \\
& \stackrel{(a)}{=} \lambda_{1} \lambda_{2} \sqrt{\frac{\alpha_{1, t} \alpha_{2, t}}{\alpha_{1, t+1} \alpha_{2, t+1}}}\left(\frac{N \rho_{t}-\operatorname{sgn}\left(\rho_{t}\right) \sqrt{P_{1} P_{2}}\left(1-\rho_{t}^{2}\right)}{P_{1}+P_{2}+2\left|\rho_{t}\right| \sqrt{P_{1} P_{2}}+N}\right) \\
& \stackrel{(b)}{=} \frac{N \rho_{t}-\operatorname{sgn}\left(\rho_{t}\right) \sqrt{P_{1} P_{2}}\left(1-\rho_{t}^{2}\right)}{\sqrt{\left(N+P_{1}\left(1-\rho_{t}^{2}\right)\right)\left(N+P_{1}\left(1-\rho_{t}^{2}\right)\right)}} \quad \forall t \geq 2, \text { (13) }
\end{aligned}
$$

where (a) follows from (12); and (b) follows from (10) and (11). It has been shown in [14] that for (13) there exists a $\rho^{\star}$ such that if $\rho_{t}=\rho^{\star}$ then $\rho_{t+k}=(-1)^{k} \rho^{\star}$ for all $k \geq 0$, where $\rho^{\star}$ is the root in the open interval $(0,1)$ of the following fourth order polynomial.

$$
\begin{aligned}
\left(P_{1}\left(1-\rho^{2}\right)+N\right) & \left(P_{2}\left(1-\rho^{2}\right)+N\right)= \\
& \left(P_{1}+P_{2}+2 \rho \sqrt{P_{1} P_{2}}+N\right) N .
\end{aligned}
$$

If we modify the control actions such that $\rho_{2}$ becomes equal to $\rho^{\star}$ instead of zero, then $\rho_{t}$ will be equal to $(-1)^{t} \rho^{\star}$ for all $t \geq 2$. Suppose in the time step $t=1$ the controller takes the actions $U_{1,1}=m$ and $U_{2,1}=-\lambda_{2} \hat{X}_{2,1}+m$, where $m$ is a Gaussian variable with zero mean and variance $\sigma_{m}^{2}$. By varying $\sigma_{m}^{2}$ the correlation coefficient $\rho_{2}$ can be made equal to any value between zero and one. Therefore by choosing $\sigma_{m}^{2}$ such that $\rho_{2}=\rho^{\star}$, we can rewrite (10) and (11) as

$$
\begin{aligned}
\alpha_{i, t} & =\alpha_{i, 2}\left(\lambda_{i}^{2} \frac{N+P_{i}\left(1-\rho^{\star}\right)}{P_{1}+P_{2}+2\left|\rho^{\star}\right| \sqrt{P_{1} P_{2}}+N}\right)^{t-2} \\
& =\alpha_{i, 2}\left(\lambda_{i}^{2} \frac{N}{N+P_{i}\left(1-\rho^{\star}\right)}\right)^{t-2},
\end{aligned}
$$

where the last equality follows from (14). We observe from (15) that $\alpha_{i, t} \rightarrow 0$ as $t \rightarrow \infty$ if

$$
\begin{aligned}
& \left(\lambda_{i}^{2} \frac{N}{N+P_{i}\left(1-\rho^{\star}\right)}\right)<1 \\
& \Rightarrow \log \left(\lambda_{i}\right)<\frac{1}{2} \log \left(1+\frac{P_{i}\left(1-\rho^{\star 2}\right)}{N}\right),
\end{aligned}
$$

which completes the proof.

\section{B. Proof of Theorem 5}

The communication and control scheme for the white Gaussian broadcast channel is in principle similar to that of the multiple-access channel where in the beginning the 
encoder separately transmit the states of the two plants in order to make them Gaussian. Thereafter the Gaussian distributed states are transmitted jointly. This scheme works as follows.

Initial time steps, $t=0,1$ : In the first two time steps the encoder transmits state observations of each plant separately. These separate initial transmissions make plant states Gaussian distributed regardless of the distribution of their initial states. However if the initial states are already Gaussian, then the following separate initial transmissions are not needed.

At time step $t=0$ the encoder ignores $X_{2,0}$ and transmits $X_{1,0}$ as $S_{0}=\sqrt{\frac{P}{\alpha_{1,0}}} X_{1,0}$. The decoder $\mathcal{D}_{1}$ receives $R_{1,0}=$ $S_{0}+Z_{0}+Z_{1,0}$. It then estimates $X_{1,0}$ as

$$
\hat{X}_{1,0}=\sqrt{\frac{\alpha_{1,0}}{P}} R_{1,0}=X_{1,0}+\sqrt{\frac{\alpha_{1,0}}{P}}\left(Z_{0}+Z_{1,0}\right) .
$$

The controller $\mathcal{C}_{1}$ then takes an action $U_{1,0}=-\lambda_{1} \hat{X}_{1,0}$ for the plant 1 , which results in $X_{1,1}=\lambda_{1}\left(X_{1,0}-\hat{X}_{1,0}\right)$. The state $X_{1,1} \sim \mathcal{N}\left(0, \alpha_{1,1}\right)$ with $\alpha_{1,1}=\lambda_{1}^{2} \frac{\alpha_{1,0}\left(N+N_{1}\right)}{P}$. The controller $\mathcal{C}_{2}$ does not take any action for the plant 2 , therefore $X_{2,1}=\lambda_{2} X_{2,0}$ with $\alpha_{2,1}=\lambda_{2}^{2} \alpha_{2,0}$.

At time step $t=1$ the encoder $\mathcal{E}$ ignores $X_{1,1}$ and transmits only $X_{2,1}$, i.e., $S_{1}=\sqrt{\frac{P}{\alpha_{2,1}}} X_{2,1}$. The decoder $\mathcal{D}_{2}$ receives $R_{2,1}=S_{1}+Z_{1}+Z_{2,1}$. It then estimates $X_{2,1}$ as

$$
\hat{X}_{2,1}=\sqrt{\frac{\alpha_{2,1}}{P}} R_{2,1}=X_{2,1}+\sqrt{\frac{\alpha_{2,1}}{P}}\left(Z_{1}+Z_{2,1}\right) .
$$

The controller $\mathcal{C}_{2}$ then takes an action $U_{2,1}=-\lambda_{2} \hat{X}_{2,1}$ for the plant 2, which results in $X_{2,2}=\lambda_{2}\left(X_{2,1}-\hat{X}_{2,1}\right)$. The state variable $X_{2,2} \sim \mathcal{N}\left(0, \alpha_{2,2}\right)$. The controller $\mathcal{C}_{1}$ does not take any action for the plant 1 , i.e., $U_{1,1}=0$, therefore $X_{1,2}=\lambda_{1} X_{1,1}$ and $X_{1,2} \sim \mathcal{N}\left(0, \alpha_{1,2}\right)$.

Similar to the multiple-access channel, the states $X_{1,2}$ and $X_{2,2}$ are now zero mean Gaussian variables with correlation coefficient $\rho_{2}=\frac{\mathbb{E}\left[X_{1,2} X_{2,2}\right]}{\sqrt{\alpha_{1,2} \alpha_{2,2}}}$ equal to zero ${ }^{2}$. Henceforth the encoder will serve both plants simultaneously.

Further time steps, $t \geq 2$ : The encoder $\mathcal{E}$ observes $X_{1, t}$ and $X_{2, t}$, and it transmits

$$
S_{t}=\sqrt{\frac{P}{D_{t}}}\left(\frac{X_{1, t}}{\sqrt{\alpha_{1, t}}}+g \frac{X_{2, t}}{\sqrt{\alpha_{2, t}}} \operatorname{sgn}\left(\rho_{t}\right)\right),
$$

where $D_{t}=1+g^{2}+2 g\left|\rho_{t}\right|, g \geq 0, \rho_{t}=$ $\frac{\mathbb{E}\left[\left(X_{1, t}-\mathbb{E}\left[X_{1, t}\right]\right)\left(X_{2, t}-\mathbb{E}\left[X_{2, t}\right]\right)\right]}{\sqrt{\alpha_{1, t} \alpha_{2, t}}}$, and $\operatorname{sgn}\left(\rho_{t}\right)=1$ if $\rho_{t} \geq 0$ and $\operatorname{sgn}\left(\rho_{t}\right)=-1$ if $\rho_{t}<0$.

The decoder $\mathcal{D}_{i}$ receives $R_{i, t}=S_{t}+Z_{t}+Z_{i, t}$. It then computes an MMSE estimate of the state of the plant $i$ as

$$
\begin{aligned}
\hat{X}_{i, t} & =\mathbb{E}\left[X_{i, t} \mid\left\{R_{i, k}\right\}_{k=0}^{t}\right] \\
& \stackrel{(a)}{=} \mathbb{E}\left[X_{i, t} \mid R_{i, t}\right] \stackrel{(b)}{=} \frac{\mathbb{E}\left[R_{i, t} X_{i, t}\right]}{\mathbb{E}\left[R_{i, t}^{2}\right]} R_{i, t},
\end{aligned}
$$

\footnotetext{
${ }^{2}$ The states in the second time step become uncorrelated irrespective of the value of the correlation between the initial states. This scheme does not exploit correlation between the initial states and thus the stability region obtained is independent of the correlation of the initial states.
}

where (a) follows from $\mathbb{E}\left[X_{i, t} R_{i, k}\right]=0$ for all $k<t$ and $X_{i, t}$ and $R_{i, t}$ are Gaussian variables; (b) follows from the fact that the optimum MMSE of the Gaussian variable is linear [20]; and we have

$$
\begin{aligned}
\mathbb{E}\left[X_{1, t} R_{1, t}\right] & =\sqrt{\frac{P \alpha_{1, t}}{D_{t}}}\left(1+g\left|\rho_{t}\right|\right) \\
\mathbb{E}\left[X_{2, t} R_{2, t}\right] & =\sqrt{\frac{P \alpha_{2, t}}{D_{t}}}\left(\rho_{t}+g \operatorname{sgn}\left(\rho_{t}\right)\right) \\
\mathbb{E}\left[R_{i, t}^{2}\right] & =P+N+N_{i} .
\end{aligned}
$$

The controller $\mathcal{C}_{i}$ takes an action $U_{i, t}=-\lambda_{i} \hat{X}_{i, t}$ for the plant $i$, which results in $X_{i, t+1}=\lambda_{i}\left(X_{i, t}-\hat{X}_{i, t}\right)$. The mean values of the states are

$$
\begin{aligned}
\mathbb{E}\left[X_{i, t+1}\right] & =\mathbb{E}\left[\lambda_{i}\left(X_{i, t}-\hat{X}_{i, t}\right)\right] \\
& \stackrel{(a)}{=} \lambda_{i} \mathbb{E}\left[X_{i, t}-\frac{\mathbb{E}\left[R_{i, t} X_{i, t}\right]}{\mathbb{E}\left[R_{i, t}^{2}\right]} R_{i, t}\right] \stackrel{(b)}{=} 0
\end{aligned}
$$

where ( $a$ ) follows from (17); and (b) follows from $\mathbb{E}\left[X_{i, 2}\right]=$ 0 and by recursively using $(a)$. The variance of the state $X_{i, t+1}$ is given by

$$
\begin{aligned}
\alpha_{i, t+1} & \triangleq \mathbb{E}\left[X_{i, t+1}^{2}\right]=\lambda_{i}^{2} \mathbb{E}\left[\left(X_{i, t}-\hat{X}_{i, t}\right)^{2}\right] \\
& =\lambda_{i}^{2}\left(\mathbb{E}\left[X_{i, t}^{2}\right]-\frac{\left(\mathbb{E}\left[R_{i, t} X_{i, t}\right)^{2}\right]}{\mathbb{E}\left[R_{i, t}^{2}\right]}\right) .
\end{aligned}
$$

By using (18) in (19) we get the following recursive equations.

$$
\begin{aligned}
& \alpha_{1, t+1}=\alpha_{1, t} \lambda_{1}^{2}\left(\frac{D_{t}\left(N+N_{1}\right)+g^{2} P\left(1-\rho_{t}^{2}\right)}{D_{t}\left(P+N+N_{1}\right)}\right) \\
& \alpha_{2, t+1}=\alpha_{2, t} \lambda_{2}^{2}\left(\frac{D_{t}\left(N+N_{2}\right)+P\left(1-\rho_{t}^{2}\right)}{D_{t}\left(P+N+N_{2}\right)}\right) .
\end{aligned}
$$

The cross-correlation between the states is given by

$$
\begin{gathered}
\mathbb{E}\left[X_{1, t+1} X_{2, t+1}\right]=\mathbb{E}\left[\lambda_{1}\left(X_{1, t}-\hat{X}_{1, t}\right) \lambda_{2}\left(X_{2, t}-\hat{X}_{2, t}\right)\right] \\
=\lambda_{1} \lambda_{2}\left(\mathbb{E}\left[X_{1, t} X_{2, t}\right]-2 \mathbb{E}\left[\hat{X}_{1, t} X_{2, t}\right]+\mathbb{E}\left[\hat{X}_{1, t} \hat{X}_{2, t}\right]\right) \\
\stackrel{(a)}{=} \lambda_{1} \lambda_{2}\left(\frac{\mathbb{E}\left[X_{1, t} X_{2, t}\right] \Pi-\mathbb{E}\left[R_{1, t} X_{1, t}\right] \mathbb{E}\left[R_{2, t} X_{2, t}\right] \Sigma}{\Pi}\right) \\
\stackrel{(b)}{=} \lambda_{1} \lambda_{2} \sqrt{\alpha_{1, t} \alpha_{2, t}}\left(\rho_{t}\right. \\
\left.\quad-\frac{P}{D_{t} \Pi}\left(\rho_{t}+g\left|\rho_{t}\right| \rho_{t}+g \operatorname{sgn}\left(\rho_{t}\right)+g \rho_{t}\right) \Sigma\right)
\end{gathered}
$$

where ( $a$ ) follows from $\mathbb{E}\left[\hat{X}_{1, t} X_{2, t}\right]=\frac{\mathbb{E}\left[R_{1, t} X_{1, t}\right] \mathbb{E}\left[R_{2, t} X_{2, t}\right]}{P+N+N_{1}}$, $\mathbb{E}\left[X_{1, t} \hat{X}_{2, t}\right]=\frac{\mathbb{E}\left[R_{1, t} X_{1, t}\right] \mathbb{E}\left[R_{2, t} X_{2, t}\right]}{P+N+N_{2}}, \quad \mathbb{E}\left[\hat{X}_{1, t} \hat{X}_{2, t}\right]=$ $\frac{\mathbb{E}\left[R_{1, t} X_{1, t}\right] \mathbb{E}\left[R_{2, t} X_{2, t}\right](P+N)}{\left(P+N+N_{1}\right)\left(P+N+N_{2}\right)}, \Pi \triangleq\left(P+N+N_{1}\right)\left(P+N+N_{2}\right)$, and $\Sigma \triangleq\left(P+N+N_{1}+N_{2}\right)$; and (b) follows from (18). Now we can write a recursive equation for the correlation coefficient $\rho_{t}$ by using (20), (21) and (22), as

$$
\begin{aligned}
& \rho_{t+1}=\frac{\mathbb{E}\left[X_{1, t+1} X_{2, t+1}\right]}{\sqrt{\alpha_{1, t+1} \alpha_{2, t+1}}}=\left(D_{t}\left(N \Sigma+N_{1} N_{2}\right) \rho_{t}-g P \Sigma\left(1-\rho_{t}^{2}\right) \operatorname{sgn}\left(\rho_{t}\right)\right) \\
& \times\left(\Pi\left(D_{t}\left(N+N_{1}\right)+g^{2} P\left(1-\rho_{t}^{2}\right)\right)\left(D_{t}\left(N+N_{2}\right)+g^{2} P\left(1-\rho_{t}^{2}\right)\right)\right)^{-\frac{1}{2}}
\end{aligned}
$$


It has been shown in [15] that for the above recursive equation there exists a $\rho^{\star}$ such that if $\rho_{t}=\rho^{\star}$ then $\rho_{t+k}=$ $(-1)^{k} \rho^{\star}$ for all $k \geq 0$, where $\rho^{\star}$ is the largest root in the open interval $(0,1)$ of the polynomial given in $(5)$. If we modify our encoding scheme such that $\rho_{2}$ becomes equal to $\rho^{\star}$ instead of zero, then $\rho_{t}$ will be equal to $(-1)^{t} \rho^{\star}$ for all $t \geq 2$. Suppose in the initial transmissions (i.e., $t=$ $0,1)$ the encoder transmits $S_{0}=\sqrt{\frac{P}{\alpha_{1,0}}} X_{1,0}+m$ and $S_{1}=$ $\sqrt{\frac{P}{\alpha_{2,1}}} X_{2,1}+m$, where $m$ is a Gaussian variable with zero mean and variance $\sigma_{m}^{2}$. In this way $\rho_{2}$ can take on value between zero and one by varying $\sigma_{m}^{2}$. Thus by choosing $\sigma_{m}^{2}$ such that $\rho_{2}=\rho^{\star}$, we can rewrite (10) and (11) as

$$
\begin{aligned}
& \alpha_{1, t}=\alpha_{1,2}\left(\lambda_{1}^{2} \frac{D^{\star}\left(N+N_{1}\right)+g^{2} P\left(1-\rho^{\star 2}\right)}{D^{\star}\left(P+N+N_{1}\right)}\right)^{t-2} \\
& \alpha_{2, t}=\alpha_{2,2}\left(\lambda_{2}^{2} \frac{D^{\star}\left(N+N_{2}\right)+P\left(1-\rho^{\star 2}\right)}{D^{\star}\left(P+N+N_{2}\right)}\right)^{t-2}
\end{aligned}
$$

Although in the modified encoding scheme we have violated the average power constraint for the first two transmissions, its effect can be neglected for infinite time horizon. We observe from (23) that $\alpha_{1, t} \rightarrow 0$ as $t \rightarrow \infty$ if

$$
\begin{aligned}
& \left(\lambda_{1}^{2} \frac{D^{\star}\left(N+N_{1}\right)+g^{2} P\left(1-\rho^{\star 2}\right)}{D^{\star}\left(P+N+N_{1}\right)}\right)<1 \\
& \Rightarrow \log \left(\lambda_{1}\right)<\frac{1}{2} \log \left(\frac{D^{\star}\left(P+N+N_{1}\right)}{D^{\star}\left(N+N_{1}\right)+g^{2} P\left(1-\rho^{\star 2}\right)}\right) .
\end{aligned}
$$

Similarly it follows from (24) that $\alpha_{2, t} \rightarrow 0$ as $t \rightarrow \infty$ if

$$
\begin{gathered}
\log \left(\lambda_{2}\right)<\frac{1}{2} \log \left(\frac{D^{\star}\left(P+N+N_{2}\right)}{D^{\star}\left(N+N_{2}\right)+P\left(1-\rho^{\star 2}\right)}\right) \\
\text { V. CONCLUSION }
\end{gathered}
$$

We study the problem of mean square stabilizing two discrete time scalar LTI systems in closed-loop via control over white Gaussian multiple-access and broadcast communication channels. We propose to use simple linear communication and control schemes which whiten the state process and make it Gaussian, and therefore the optimal decoding of the transmitted state values at the remote control unit(s) is linear and memoryless. These schemes are adaptations of the coding schemes introduced by Ozarow and Leung, which provide double exponential error decay and therefore second moment stability is achieved. The stability regions obtained for the control over multiple-access and the broadcast communication channels are associated with the achievable rate regions for the given channels with noiseless feedback. Therefore our results reveal relationship between mean square stability of the two plants and the communication channels' parameters, i.e., average power consumed by the encoder(s) and the average power of the noise components in different links.

The stability results provided in this paper can be easily extended for the setup where the links from the controller(s) to the plants are also white Gaussian communication channels. For this setup we can have an encoder at each control unit to encode the control action and an MMSE decoder at each plant to decode the transmitted value of the control action. As long as the encoders, the decoders, and the controllers are linear, the nature of the problem does not change and the stability results can be easily obtained [12].

\section{ACKNOWLEDGEMENT}

The authors wish to thank Serdal Yüksel of Queen's University in Canada for fruitful discussions and valuable suggestions that helped to improve the quality of this submission.

\section{REFERENCES}

[1] R. Bansal and T. Basar, "Simultaneous design of measurement and control strategies for stochastic systems with feedback," Automatica, vol. 25 , no. 5, pp. 679-694, 1989.

[2] N. Elia, "When Bode meets Shannon: control-oriented feedback communication schemes," IEEE Trans. on Automat. Control, vol. 49, no. 9, pp. 1477-1488, 2004.

[3] G. N. Nair and R. J. Evans, "Mean square stabilisability of stochastic linear systems with data rate constraints," in Proc. IEEE CDC, 2002.

[4] _ "Stabilizability of stochastic linear systems with finite feedback data rates," SIAM J. Control Optim., vol. 43, no. 2, pp. 413-436, 2004.

[5] S. Tatikonda and S. Mitter, "Control under communication constraints," IEEE Trans. Automat. Control, vol. 49, no. 7, pp. 1056-1068, 2004.

[6] _ "Control over noisy channels," IEEE Trans. Automat. Control, vol. 49, no. 7, pp. 1196-1201, 2004.

[7] A. Matveev and A. Savkin, "An analogue of shannon information theory for detection and stabilization via noisy discrete communication channels," SIAM J. Control Optim., vol. 46, no. 4, pp. 1323-1367, 2007.

[8] A. Sahai and S. Mitter, "The necessity and sufficiency of anytime capacity for stabilization of a linear system over noisy communication links-part i: Scalar systems," IEEE Trans. Inform. Theory, vol. 52, no. 8, pp. 3369-3395, 2006.

[9] E. Silva, M. Derpich, J. Ostergaard, and D. Quevedo, "Simple coding for achieving mean square stability over bit-rate limited channels," in Proc. IEEE CDC, 2008.

[10] J. Freudenberg, R. Middleton, and V. Solo, "Stabilization and disturbance attenuation over a Gaussian communication channel," IEEE Trans. Automat. Control, vol. 55, no. 3, pp. 795-799, 2010.

[11] R. Middleton, A. Rojas, J. Freudenberg, and J. Braslavsky, "Feedback stabilization over a first order moving average gaussian noise channel," IEEE Trans. Automat. Control, vol. 54, no. 1, 2009.

[12] S. Yüksel and T. Basar, "Achievable rates for stability of LTI systems over noisy forward and feedback channels," in Proc. Conference on Information Sciences and Systems, 2005.

[13] T. Cover and J. Thomas, Elements of information theory. John Wiley Sons, Inc., 2006.

[14] L. H. Ozarow, "The capacity of the white gaussian multiple-access channel with feed-back," IEEE Trans. Inform. Theory, vol. 30, no. 4, pp. 623-629, July 1984.

[15] L. Ozarow and S. Leung-Yan-Cheong, "An achievable region and outer bound for the gaussian broadcast channel with feedback," IEEE Trans. Inform. Theory, vol. 30, no. 4, pp. 667-671, July 1984.

[16] Schalkwijk and T. Kailath, "A coding scheme for additive noise channels with feedback-i: No bandwidth constraint," IEEE Trans. Inform. Theory, vol. 12, no. 2, pp. 172-182, 1966.

[17] A. A. Zaidi, T. J. Oechtering, and M. Skoglund, "Rate sufficient conditions for closed-loop control over AWGN relay channels," in IEEE ICCA, 2010.

[18] A. A. Zaidi, T. J. Oechtering, M. Skoglund, and S. Yüksel, "Closedloop control over half-duplex AWGN relay channels," in Reglermöte, 2010.

[19] J. Freudenberg, R. Middleton, and V. Solo, "The minimal signal-tonoise ratio rqeuired to stabilize over a noisy channel," in Proc. ACC, 2006.

[20] M. Hayes, Statistical digital signal processing and modelling. John Wiley Sons, Inc., 1996. 